\title{
On Hardy spaces associated with certain Schrödinger operators in dimension 2
}

\author{
Jacek Dziubański and Jacek Zienkiewicz
}

\begin{abstract}
We study the Hardy space $H^{1}$ associated with the Schrödinger operator $L=-\Delta+V$ on $\mathbb{R}^{2}$, where $V \geq 0$ is a compactly supported nonzero $C^{2}$-potential. We prove that this space, which is originally defined by means of the maximal function associated with the semigroup generated by $-L$, admits a special atomic decomposition with atoms satisfying a weighted cancellation condition with a weight of logarithmic growth.
\end{abstract}

\section{Introduction}

The aim of this paper is to prove a special atomic characterization of the Hardy spaces $H^{1}$ associated with Schrödinger operators in $\mathbb{R}^{2}$ with compactly supported non-negative smooth potentials. In other dimensions such characterizations were obtained in [4] and [10], and only the 2-dimensional situation remained open.

Let $K_{t}(x, y)$ be the integral kernels of the semigroup of linear operators $\left\{K_{t}\right\}_{t>0}$ on $\mathbb{R}^{2}$, generated by a Schrödinger operator $-L=\Delta-V(x)$, where $V(x)$ is a non-zero nonnegative $C^{2}$-function supported by the unit open ball $B(0,1)$. The Feynman-Kac formula

$$
K_{t} f(x)=\mathrm{E}^{x}\left(e^{-\int_{0}^{t} V\left(X_{s}\right) d s} f\left(X_{t}\right)\right),
$$

where $X_{t}$ is the Brownian motion associated with the heat semigroup $P_{t}:=e^{t \Delta}$ (see, e.g., Chapter V of [16]), implies that

$$
0 \leq K_{t}(x, y) \leq(4 \pi t)^{-1} \exp \left(-|x-y|^{2} / 4 t\right):=P_{t}(x-y) .
$$

Clearly, for every $1 \leq p<\infty$, the family $\left\{K_{t}\right\}_{t>0}$ forms a semigroup of linear contractions on $L^{p}\left(\mathbb{R}^{2}\right)$.

The Hardy space $H_{L}^{1}$ is defined by means of the maximal function for the semigroup $\left\{K_{t}\right\}_{t>0}$, namely,

$$
H_{L}^{1}=\left\{f \in L^{1}\left(\mathbb{R}^{2}\right): \mathcal{M}_{L} f \in L^{1}\left(\mathbb{R}^{2}\right)\right\},
$$

Mathematics Subject Classification (2010): Primary 42B30, 35J10; Secondary 42B35.

Keywords: Hardy spaces, maximal functions, Schrödinger operator. 
where

$$
\mathcal{M}_{L} f(x)=\sup _{t>0}\left|K_{t} f(x)\right|
$$

The norm $\|f\|_{H_{L}^{1}}$ on the space $H_{L}^{1}$ is given by the formula

$$
\|f\|_{H_{L}^{1}}=\left\|\mathcal{M}_{L} f\right\|_{L^{1}\left(\mathbb{R}^{2}\right)}
$$

Let $w(x)>0$ be a locally integrable function. We say that a measurable function $b$ defined on $\mathbb{R}^{2}$ is an atom for the Hardy space $H_{w \text {, at }}^{1}$ if there exists a ball $B=B\left(x_{0}, r\right)$ such that

$$
\begin{gathered}
\operatorname{supp} b \subset B, \quad\|b\|_{L^{\infty}} \leq|B|^{-1}, \\
\int_{\mathbb{R}^{2}} b(x) w(x) d x=0 .
\end{gathered}
$$

The atomic norm $\|f\|_{H_{w, \text { at }}^{1}}$ is given by the formula

(1.8) $\|f\|_{H_{w, \text { at }}^{1}}=\inf \left\{\sum_{j}\left|\lambda_{j}\right|: f=\sum_{j} \lambda_{j} b_{j}, \lambda_{j} \in \mathbb{C}, b_{j}\right.$ are atoms for $\left.H_{w, \text { at }}^{1}\right\}$.

Clearly, if $w \equiv 1$, then the $H_{w \text {, at }}^{1}$-atoms coincide with the classical $(1, \infty)$-atoms for the Hardy space $H^{1}\left(\mathbb{R}^{2}\right)$. For the theory of the classical real Hardy spaces $H^{p}\left(\mathbb{R}^{d}\right)$ we refer the reader to [3], [11], [12], [17], and the references therein.

Our goal in this paper is to prove the following theorem:

Theorem 1.1. Assume that $L=-\Delta+V$ is a Schrödinger operator in $\mathbb{R}^{2}$, where $V$ is a non-zero nonnegative $C^{2}$-function such that $\operatorname{supp} V$ is contained in the unit ball $B(0,1)$. Then there exists a regular $L$-harmonic weight $w$ such that

$$
C^{-1} \ln (2+|x|) \leq w(x) \leq C \ln (2+|x|),
$$

$H_{L}^{1}=H_{w, \text { at }}^{1}$, and

$$
C^{-1}\|f\|_{H_{w, \text { at }}^{1}} \leq\|f\|_{H_{L}^{1}} \leq C\|f\|_{H_{w, \text { at }}^{1}}
$$

The construction and the properties of the weight $w$ are given in Section 3.

Let us finally emphasize the differences which occur in atomic decompositions of the Hardy spaces associated with Schrödinger operators with compactly supported potentials in dimensions different than 2. It was proved in [10] that for $d \geq 3$ and any compactly supported nonnegative $V \in L^{q}\left(\mathbb{R}^{d}\right), q>d / 2$, the Hardy space $H_{-\Delta+V}^{1}$ coincides with $H_{w \text {, at }}^{1}$, where

$$
w(x)=\lim _{t \rightarrow \infty} \int_{\mathbb{R}^{d}} K_{t}(x, y) d y .
$$


Here $K_{t}(x, y)$ are the integral kernels of the semigroup generated by $\Delta-V$ in $\mathbb{R}^{d}$. The function $w$ defined by (1.11) satisfies

$$
0<c \leq w(x) \leq 1, \quad \lim _{|x| \rightarrow \infty} w(x)=1, \quad\left|w(x)-w\left(x^{\prime}\right)\right| \leq C\left|x-x^{\prime}\right| .
$$

See [10] for details and proofs.

The one-dimensional situation is different and was studied in [4]. Let $V \in L_{\text {loc }}^{1}(\mathbb{R})$ be a nonnegative potential. In order to define the notion of an atom for the space $H_{-\Delta+V}^{1}$, one defines the auxiliary function

$$
\rho(y):=\inf \left\{r>0: r \int_{y-r}^{y+r} V(x) d x \geq 1\right\} .
$$

Then atoms for $H_{-\Delta+V}^{1}$ are either the classical $(1, \infty)$-atoms for the Hardy space $H^{1}(\mathbb{R})$ or $|I|^{-1} \chi_{I}(x)$, where $I=(y-\rho(y), y+\rho(y))$. Hence, in this case, the Hardy space is local, where the scale of localization is adapted to the behavior of the potential (see [4]).

The reader interested in results concerning Hardy spaces associated with semigroups of linear operators, and in particular semigroups generated by Schrödinger operators, is referred to [1], [2], [4], [6], [7], [8], [9], [10] and [15].

The paper is organized as follows. In Section 2 we derive estimates for the integral kernels $K_{t}(x, y)$ of the semigroup generated by $-L=\Delta-V$, where $V \in C^{2}(B(0,1)) \subset \mathbb{R}^{2}, V \geq 0, V \not \equiv 0$. Then we prove some properties of the operator $V L^{-1}$. Section 3 is devoted for the construction of the weight function $w$ (see Theorem 1.1). In Section 4 we provide a relation between the classical atoms and the $H_{w}^{1}$, at -atoms (see Proposition 4.2). The proofs of the inequalities in (1.10) are presented in Sections 5 and 6.

Acknowledgments. The authors want to thank the reviewer for his valuable remarks which improved the presentation of the paper.

\section{Estimates of kernels in $\mathbb{R}^{2}$}

Henceforth, unless otherwise stated, we assume that $V$ is a non-zero nonnegative $C^{2}$-potential supported in the unit ball $B(0,1)$ of $\mathbb{R}^{2}, K_{t}(x, y)$ are the integral kernels of the semigroup $\left\{K_{t}\right\}_{t>0}$ generated by $-L=\Delta-V(x)$.

For $C_{0}>0$ (big enough) we define

$$
G(x):=\frac{1}{2 \pi} \int_{\mathbb{R}^{2}} \ln |x-y| V(y) d y+C_{0} .
$$

Then $G \in C^{\infty}\left(\mathbb{R}^{2}\right)$ and, if we take $C_{0}$ large,

$$
G(x) \geq 2 \quad \text { and } \quad G(x) \sim \ln (2+2|x|) \quad \text { for } x \in \mathbb{R}^{2} .
$$


The function $G(x)$ is $L$-subharmonic, that is, $L G(x) \geq 0$. Indeed,

$$
\begin{aligned}
L G(x) & =(-\Delta+V(x))\left(\frac{1}{2 \pi} \int_{\mathbb{R}^{2}} \ln |x-y| V(y) d y+C_{0}\right) \\
& =-V(x)+V(x) G(x) \geq 0 .
\end{aligned}
$$

Hence,

$$
\frac{d}{d t} \int_{\mathbb{R}^{2}} K_{t}(z, y) G(y) d y=-\int_{\mathbb{R}^{2}} K_{t}(z, y) L G(y) d y \leq 0,
$$

and, consequently,

$$
\int_{\mathbb{R}^{2}} K_{t}(z, y) G(y) d y \leq G(z)
$$

Since $G(x)$ is bounded on compact sets,

$$
\int K_{t}(z, y) G(y) d y \leq C \text { for } t>0 \text { and }|z| \leq 3 .
$$

Lemma 2.1. There is $C>0$ such that

$$
\int_{\mathbb{R}^{2}} K_{t}(z, y) d y \leq \begin{cases}1 & \text { for } t \leq 2(1+|z|), \\ C \frac{\ln (2+2|z|)}{\ln t} & \text { for } t>2(1+|z|) .\end{cases}
$$

Proof. Thanks to (1.2), it suffices to consider $t>2(1+|z|)$. Using (2.2) together with (2.1), we have that

$$
\begin{aligned}
\int_{\mathbb{R}^{2}} K_{t}(z, y) d y & =\int_{|y| \leq t^{1 / 4}} K_{t}(z, y) d y+\int_{|y|>t^{1 / 4}} K_{t}(z, y) d y \\
& \leq C t^{-1} \int_{|y| \leq t^{1 / 4}} d y+C \int_{|y|>t^{1 / 4}} K_{t}(z, y) \frac{\ln |y|}{\ln t^{1 / 4}} d y \\
& \leq C t^{-1 / 2}+C \frac{G(z)}{\ln t^{1 / 4}} \leq C \frac{\ln (2+2|z|)}{\ln t}
\end{aligned}
$$

Lemma 2.2. There exists a constant $\delta>0$ such that

$$
\int_{\mathbb{R}^{2}} K_{t}(x, y) d y \geq \delta \frac{\ln (2+|x|)}{\ln t} \text { for } t>2(1+|x|) .
$$

Proof. The Feynman-Kac formula (1.1) implies that

$$
e^{-t\|V\|_{\infty}} P_{t}(x-y) \leq K_{t}(x, y) \text {. }
$$

Set $\phi(x, t)=\int_{\mathbb{R}^{2}} K_{t}(x, y) d y$. Clearly, by (1.2) and the semigroup property,

$$
0<\phi\left(x, t_{1}\right) \leq \phi\left(x, t_{2}\right) \text { for } t_{1}>t_{2}>0 .
$$

Let $\Omega=\left\{x \in \mathbb{R}^{2}:|x|>1\right\}$. We have

$$
0 \geq \frac{d}{d t} \phi(x, t)=\Delta \phi(x, t)-V(x) \phi(x, t)=\Delta \phi(x, t) \quad \text { for } x \in \Omega,
$$


and so for fixed $t, \phi(x, t)$ is superharmonic on $\Omega$. Moreover, from the FeynmanKac formula we conclude that there exists a constant $\delta_{0}>0$ such that $\phi(x, t) \geq \delta_{0}$ for $|x|=\sqrt{t}>2$. For fixed $t>4$, let

$$
u(x)=\delta_{0} \frac{\ln |x|}{\ln \sqrt{t}} .
$$

Then

$$
0=u(x) \leq \phi(x, t) \text { for }|x|=1 \quad \text { and } \quad \delta_{0}=u(x) \leq \phi(x, t) \text { for }|x|=\sqrt{t},
$$

and, consequently, since $u$ is harmonic,

$$
\delta_{0} \frac{\ln |x|}{\ln \sqrt{t}}=u(x) \leq \phi(x, t)=\int_{\mathbb{R}^{2}} K_{t}(x, y) d y \quad \text { for } 1 \leq|x| \leq \sqrt{t} .
$$

Now we consider $|x| \leq 2$. Let $t>5$. Then,

$$
\begin{aligned}
\int_{\mathbb{R}^{2}} K_{t}(x, y) d y & =\int_{\mathbb{R}^{2}} \int_{\mathbb{R}^{2}} K_{1}(x, z) K_{t-1}(z, y) d z d y \\
& \geq \int_{\mathbb{R}^{2}} \int_{2<|z|<3} K_{1}(x, z) K_{t-1}(z, y) d z d y \\
& \geq \inf _{\{|x| \leq 2,2<|z|<3\}}\left\{K_{1}(x, z)\right\} \int_{\mathbb{R}^{2}} \int_{2<|z|<3} K_{t-1}(z, y) d z d y \\
& \geq \frac{c \delta_{0}}{\ln \sqrt{t-1}},
\end{aligned}
$$

where in the last inequality we have used (2.5) and (2.7). Thus, thanks to (2.6) and (2.7), the lemma is proved for $t>2(1+|x|)^{2}$. Assume now that $t>2(1+|x|)$. Since $t^{2}>t$

$$
\int_{\mathbb{R}^{2}} K_{t}(x, y) d y \geq \int_{\mathbb{R}^{2}} K_{t^{2}}(x, y) d y \geq \delta \frac{\ln (2+|x|)}{\ln \left(t^{2}\right)}=\frac{\delta \ln (2+|x|)}{2 \ln t} .
$$

The perturbation formula (see, e.g., Chapter 3 of [5]) asserts that

$$
P_{t}=K_{t}+\int_{0}^{t} P_{t-s} V K_{s} d s .
$$

Thus

$$
\begin{aligned}
\int_{0}^{t} \int_{\mathbb{R}^{2}} V(z) K_{s}(z, y) d z d s & =\int_{\mathbb{R}^{2}}\left(P_{t}(x-y)-K_{t}(x, y)\right) d x \\
& =1-\int_{\mathbb{R}^{2}} K_{t}(x, y) d x .
\end{aligned}
$$

Hence, using Lemma 2.1, we get

$$
\int_{0}^{\infty} \int_{\mathbb{R}^{2}} V(z) K_{s}(z, y) d z d s=1
$$


Lemma 2.3. There exists a constant $C>0$ such that for $x, y \in \mathbb{R}^{2}$ we have

$$
\int_{2}^{\infty} K_{t}(x, y) d t \leq C \min (\ln (2+|x|), \ln (2+|y|)) .
$$

Proof. Observe first that for $x, y \in \mathbb{R}^{2}$, and $t \geq 2$ one has

$$
K_{t}(x, y) \leq C \frac{\ln (2+|x|) \ln (2+|y|)}{t(\ln t)^{2}} .
$$

Indeed, if $u \in \mathbb{R}^{2}$, then from Lemma 2.1 we conclude

$$
\begin{aligned}
K_{t}(x, u) & =\int_{\mathbb{R}^{2}} K_{t / 2}(x, z) K_{t / 2}(z, u) d z \leq C t^{-1} \int_{\mathbb{R}^{2}} K_{t / 2}(x, z) d z \\
& \leq C \frac{\ln (2+|x|)}{t \ln t} .
\end{aligned}
$$

Applying again Lemma 2.1 combined with (2.13), we obtain

$$
\begin{aligned}
K_{t}(x, y) & =\int_{\mathbb{R}^{2}} K_{t / 2}(x, z) K_{t / 2}(z, y) d z \\
& \leq \frac{C \ln (2+|x|)}{t \ln t} \int_{\mathbb{R}^{2}} K_{t / 2}(z, y) d z \leq \frac{C \ln (2+|x|) \ln (2+|y|)}{t(\ln t)^{2}} .
\end{aligned}
$$

Clearly, (2.12) implies (2.11) for $|x|,|y| \leq 2$. Thus, to complete the proof of the lemma, we can assume that $|y| \geq|x|$ and $|y|>2$. If additionally $|y|>2|x|$, then by (2.12) and (1.2),

$$
\begin{aligned}
& \int_{2}^{\infty} K_{t}(x, y) d t \leq \int_{2}^{|y|^{2}} K_{t}(x, y) d t+\int_{|y|^{2}}^{\infty} K_{t}(x, y) d t \\
& \quad \leq C \int_{2}^{|y|^{2}} t^{-1} e^{-|y|^{2} / 16 t} d t+C \int_{|y|^{2}}^{\infty} \frac{\ln (2+|x|) \ln (2+|y|)}{t(\ln t)^{2}} d t \leq C \ln (2+|x|) .
\end{aligned}
$$

If $2|x| \geq|y| \geq|x|$, then from (2.12) and (1.2) we get

$$
\int_{2}^{\infty} K_{t}(x, y) d t \leq C \int_{2}^{|y|^{2}} t^{-1} d t+C \int_{|y|^{2}}^{\infty} \frac{\ln (2+|x|) \ln (2+|y|)}{t(\ln t)^{2}} d t \leq C \ln (2+|x|) .
$$

For $\varepsilon>0$ we set

$$
\Delta_{\varepsilon}:=\Delta-\varepsilon I, \quad L_{\varepsilon}:=-\Delta+\varepsilon I+V=-\Delta_{\varepsilon}+V .
$$

We have

$$
\Delta_{\varepsilon}^{-1}=-\int_{0}^{\infty} e^{-\varepsilon t} P_{t} d t, \quad L_{\varepsilon}^{-1}=\int_{0}^{\infty} e^{-\varepsilon t} K_{t} d t
$$

Since $P_{t}$ and $K_{t}$ are contractions on every $L^{p}\left(\mathbb{R}^{2}\right)$, the operators $\Delta_{\varepsilon}^{-1}$ and $L_{\varepsilon}^{-1}$ are bounded on every $L^{p}\left(\mathbb{R}^{2}\right), 1 \leq p<\infty$. Moreover,

$$
\left(I-V \Delta_{\varepsilon}^{-1}\right)\left(I-V L_{\varepsilon}^{-1}\right)=\left(I-V L_{\varepsilon}^{-1}\right)\left(I-V \Delta_{\varepsilon}^{-1}\right)=I .
$$

These equalities can be proved by direct computation if we substitute $V=\Delta_{\varepsilon}+L_{\varepsilon}$. 
For $f \in L^{1}\left(\mathbb{R}^{2}\right)$ let

$$
L^{-1} f(x)=\int_{0}^{\infty} \int_{\mathbb{R}^{2}} K_{t}(x, y) f(y) d y d t .
$$

Formula (2.10) implies that the operator $V L^{-1}$ is bounded on $L^{1}\left(\mathbb{R}^{2}\right)$ (see also Lemma 2.3). Clearly,

$$
I=\left(I-V \Delta_{\varepsilon}^{-1}\right)\left(I-V L^{-1}\right)+\left(I-V \Delta_{\varepsilon}^{-1}\right)\left(V L^{-1}-V L_{\varepsilon}^{-1}\right) \quad \text { on } L^{1}\left(\mathbb{R}^{2}\right) .
$$

Finally let $\Delta^{-1} f(x)=f * E(x)$, with $E(x)=(2 \pi)^{-1} \ln |x|$. Since $E$ belongs to $\mathrm{BMO}\left(\mathbb{R}^{2}\right)$, there is a constant $C>0$ such that for any classical $H^{1}\left(\mathbb{R}^{2}\right)$-atom $a$ one has $\left|\Delta^{-1} a(x)\right| \leq C$ and

$$
\Delta^{-1} a(x)=-\lim _{\varepsilon \rightarrow 0^{+}} \int_{\varepsilon}^{1 / \varepsilon} P_{t} a(x) d t
$$

(see also (5.2) and (5.3)).

As a consequence of (2.10) we have the following lemma:

Lemma 2.4. For every $f \in L^{1}\left(\mathbb{R}^{2}\right)$

$$
\int\left(I-V L^{-1}\right) f(x) d x=0 .
$$

For $\varepsilon>0$ let

$$
W_{\varepsilon}(x, y):=V(x) \int_{0}^{\infty} K_{t}(x, y)\left(1-e^{-\varepsilon t}\right) d t .
$$

It is clear that $W_{\varepsilon}(x, y)$ is the integral kernel of the operator $V L^{-1}-V L_{\varepsilon}^{-1}$.

Lemma 2.5. As $\varepsilon \rightarrow 0, W_{\varepsilon}(x, y) \rightarrow 0$ uniformly on sets of the form $\mathbb{R}^{2} \times B(0, R)$.

Proof. Recall that $V$ is a $C^{2}$ function supported in $B(0,1)$. Hence, $W_{\varepsilon}(x, y)=0$ for $|x| \geq 1$. Fix $R>2$. If $|y| \leq R$ and $|x|<1$, then by (1.2) and (2.12) we obtain

$$
\begin{aligned}
W_{\varepsilon}(x, y) & \leq V(x) \int_{0}^{R}\left\|K_{t}\right\|_{\infty} \varepsilon t d t+V(x) \int_{R}^{\infty} K_{t}(x, y)\left(1-e^{-\varepsilon t}\right) d t \\
& \leq C\|V\|_{\infty} R \varepsilon+C\|V\|_{\infty} \int_{R}^{\infty} \frac{\ln (2+R)}{t(\ln t)^{2}}\left(1-e^{-\varepsilon t}\right) d t
\end{aligned}
$$

The lemma follows by applying the Lebesgue dominated convergence theorem.

The following corollary can be concluded from Lemmas 2.3 and 2.5:

Corollary 2.6. $\left\|V L^{-1}-V L_{\varepsilon}^{-1}\right\|_{L^{1} \rightarrow L^{1}} \leq 1$. Moreover, for every $f \in L^{1}\left(\mathbb{R}^{2}\right)$,

$$
\lim _{\varepsilon \rightarrow 0}\left\|\left(V L^{-1}-V L_{\varepsilon}^{-1}\right) f\right\|_{L^{1}\left(\mathbb{R}^{2}\right)}=0 .
$$




\section{Auxiliary weight function}

Let

$$
\theta(\varepsilon)=\frac{1}{4 \pi} \int_{1}^{\infty} \exp (-\varepsilon u) \frac{d u}{u}=\frac{1}{4 \pi} \int_{\varepsilon}^{\infty} \exp (-u) \frac{d u}{u}
$$

Note that

$$
\theta(\varepsilon)-\frac{1}{4 \pi} \ln \frac{1}{\varepsilon}=c_{1}+O(\varepsilon) \quad \text { as } \varepsilon \rightarrow 0^{+} .
$$

For $0<\varepsilon \leq 1$ we define the weight function

$$
w_{\varepsilon}(y)=\theta(\varepsilon) \int_{\mathbb{R}^{2}} \int_{0}^{\infty} V(z) K_{s}(z, y)\left(1-e^{-\varepsilon s}\right) d s d z
$$

Proposition 3.1. There are constants $C, c>0$ such that

$$
\begin{aligned}
w_{\varepsilon}(y) & \leq C \ln (2+2|y|), \\
c \ln (2+2|y|) & \leq w_{\varepsilon}(y) \quad \text { for }|y|<\varepsilon^{-1} .
\end{aligned}
$$

Proof. We split the integral which defines $w_{\varepsilon}$ into two parts:

$$
\begin{aligned}
w_{\varepsilon}(y)= & \theta(\varepsilon) \int_{0}^{\varepsilon^{-1}} \int_{\mathbb{R}^{2}} V(z) K_{s}(z, y)\left(1-e^{-\varepsilon s}\right) d z d s \\
& +\theta(\varepsilon) \int_{\varepsilon^{-1}}^{\infty} \int_{\mathbb{R}^{2}} V(z) K_{s}(z, y)\left(1-e^{-\varepsilon s}\right) d z d s \\
:= & J_{1}^{\varepsilon}(y)+J_{2}^{\varepsilon}(y) .
\end{aligned}
$$

Using (2.10) and Lemma 2.1, we get

$$
\begin{aligned}
J_{2}^{\varepsilon}(y) & \sim \theta(\varepsilon) \int_{\varepsilon^{-1}}^{\infty} \int_{\mathbb{R}^{2}} V(z) K_{s}(z, y) d z d s \\
& =\theta(\varepsilon)\left(\int_{0}^{\infty} \int_{\mathbb{R}^{2}} V(z) K_{s}(z, y) d z d s-\int_{0}^{\varepsilon^{-1}} \int_{\mathbb{R}^{2}} V(z) K_{s}(z, y) d z d s\right) \\
& =\theta(\varepsilon)\left(1-\left(1-\int_{\mathbb{R}^{2}} K_{\varepsilon^{-1}}(z, y) d z\right)\right) \leq C \theta(\varepsilon) \frac{\ln (2+2|y|)}{\ln 1 / \varepsilon} \\
& \leq C \ln (2+2|y|) .
\end{aligned}
$$

Observe that if $|y|<\varepsilon^{-1}$, then from Lemma 2.2 we obtain

$$
J_{2}^{\varepsilon}(y) \geq c \ln (2+|y|)
$$

We now turn to estimating $J_{1}^{\varepsilon}(y)$. By the perturbation formula (see (2.9)) 
and Lemma 2.1 we have

$$
\begin{aligned}
J_{1}^{\varepsilon}(y)= & \theta(\varepsilon)\left(\int_{0}^{\varepsilon^{-1}} \int_{\mathbb{R}^{2}} V(z) K_{s}(z, y) d z d s\right. \\
& \left.-\int_{0}^{\varepsilon^{-1}} \int_{\mathbb{R}^{2}}(\varepsilon+V(z)) K_{s}(z, y) e^{-\varepsilon s} d z d s+\int_{0}^{\varepsilon^{-1}} \int_{\mathbb{R}^{2}} \varepsilon K_{s}(z, y) e^{-\varepsilon s} d z d s\right) \\
= & \theta(\varepsilon)\left(-\int_{\mathbb{R}^{2}} K_{\varepsilon^{-1}}(z, y) d z\right. \\
& \left.+\int_{\mathbb{R}^{2}} K_{\varepsilon^{-1}}(z, y) e^{-1} d z+\int_{0}^{\varepsilon^{-1}} \int_{\mathbb{R}^{2}} \varepsilon K_{s}(z, y) e^{-\varepsilon s} d z d s\right) \\
= & \theta(\varepsilon)\left(e^{-1}-1\right) \int_{\mathbb{R}^{2}} K_{\varepsilon^{-1}}(z, y) d z+\theta(\varepsilon) \varepsilon \int_{0}^{\varepsilon^{-1}} \int_{\mathbb{R}^{2}} K_{s}(z, y) e^{-\varepsilon s} d z d s \\
\leq & C \theta(\varepsilon) \frac{\ln (2+2|y|)}{\ln (1 / \varepsilon)}+\theta(\varepsilon) \varepsilon \int_{0}^{\varepsilon^{-1 / 2}} \int_{\mathbb{R}^{2}} K_{s}(z, y) d z d s \\
& +\theta(\varepsilon) \varepsilon \int_{\varepsilon^{-1 / 2}}^{\varepsilon^{-1}} e^{-\varepsilon s} \ln (2+2|y|)(\ln \sqrt{1 / \varepsilon})^{-1} d s \\
\leq & C \ln (2+2|y|)+C \theta(\varepsilon) \varepsilon^{1 / 2}+C \theta(\varepsilon) \varepsilon \ln (2+2|y|)(\ln 1 / \varepsilon)^{-1} \int_{\varepsilon^{-1 / 2}}^{\varepsilon^{-1}} d s \\
\leq & C \ln (2+2|y|) .
\end{aligned}
$$

Thus from (3.1)-(3.4) we get the proposition.

From Proposition 3.1 we conclude that there is a subsequence $\varepsilon_{j} \rightarrow 0^{+}$such that $w_{\varepsilon_{j}}(x)$ converges to a function $w(x)$ in the weak* sense and (1.9) holds.

Since for every $y \in \mathbb{R}^{2}$ and $t>0$ the function $x \mapsto K_{t}(x, y)$ is continuous and has Gaussian decay, we have

$$
\lim _{j \rightarrow \infty} \int_{\mathbb{R}^{2}} w_{\varepsilon_{j}}(x) K_{t}(x, y) d x=\int_{\mathbb{R}^{2}} w(x) K_{t}(x, y) d x .
$$

Proposition 3.2. The function $w$ is L-harmonic, that is,

$$
K_{t} w(y)=\int_{\mathbb{R}^{2}} w(x) K_{t}(x, y) d x=w(y) \quad \text { for every } t>0 .
$$

In order to prove the proposition we need the following lemma:

Lemma 3.3. For every $t>0$, there is a constant $C>0$ such that, for every $0<\varepsilon<1$, one has

$$
\left|K_{t} w_{\varepsilon}(x)-w_{\varepsilon}(x)\right| \leq C \theta(\varepsilon) \varepsilon
$$


Proof of Lemma 3.3. We have:

$$
\begin{aligned}
K_{t} w_{\varepsilon}(x)= & \theta(\varepsilon) \int_{\mathbb{R}^{2}} \int_{0}^{\infty} \int_{\mathbb{R}^{2}} K_{t}(x, y) V(z) K_{s}(z, y)\left(1-e^{-\varepsilon s}\right) d z d s d y \\
= & \theta(\varepsilon) \int_{0}^{\infty} \int_{\mathbb{R}^{2}} V(z) K_{s+t}(x, z)\left(1-e^{-\varepsilon s}\right) d z d s \\
= & \theta(\varepsilon) \int_{t}^{\infty} \int_{\mathbb{R}^{2}} V(z) K_{s}(x, z)\left(1-e^{\varepsilon t} e^{-\varepsilon s}\right) d z d s \\
= & \theta(\varepsilon) \int_{t}^{\infty} \int_{\mathbb{R}^{2}} V(z) K_{s}(x, z)\left(1-e^{-\varepsilon s}\right) d z d s \\
& +\theta(\varepsilon) \int_{t}^{\infty} \int_{\mathbb{R}^{2}} V(z) K_{s}(x, z)\left(e^{-\varepsilon s}-e^{\varepsilon t} e^{-\varepsilon s}\right) d z d s \\
= & \theta(\varepsilon) \int_{0}^{\infty} \int_{\mathbb{R}^{2}} V(z) K_{s}(x, z)\left(1-e^{-\varepsilon s}\right) d z d s \\
& -\theta(\varepsilon) \int_{0}^{t} \int_{\mathbb{R}^{2}} V(z) K_{s}(x, z)\left(1-e^{-\varepsilon s}\right) d z d s \\
& +\theta(\varepsilon) \int_{t}^{\infty} \int_{\mathbb{R}^{2}} V(z) K_{s}(x, z) e^{-\varepsilon s}\left(1-e^{\varepsilon t}\right) d z d s \\
= & w_{\varepsilon}(x)-\theta(\varepsilon) \int_{0}^{t} \int_{\mathbb{R}^{2}} V(z) K_{s}(x, z)\left(1-e^{-\varepsilon s}\right) d z d s . \\
& +\theta(\varepsilon) \int_{t}^{\infty} \int_{\mathbb{R}^{2}} V(z) K_{s}(x, z) e^{-\varepsilon s}\left(1-e^{\varepsilon t}\right) d z d s
\end{aligned}
$$

which, by using (2.10) and the mean value theorem, implies the lemma.

Proof of Proposition 3.2. Write $v(x)=K_{t} w(x)$. Since $w(y)$ is the weak* limit of $w_{\varepsilon_{j}}(y)$, we get

$$
\lim _{j \rightarrow \infty} \int_{\mathbb{R}^{2}} w_{\varepsilon_{j}}(y) K_{t}(x, y) d y=\int_{\mathbb{R}^{2}} w(y) K_{t}(x, y) d y=v(x) .
$$

On the other hand, by Lemma 3.3, we have

$$
v(x)=\lim _{j \rightarrow \infty} \int_{\mathbb{R}^{2}} w_{\varepsilon_{j}}(y) K_{t}(x, y) d y=\lim _{j \rightarrow \infty} w_{\varepsilon_{j}}(x)+O\left(\theta\left(\varepsilon_{j}\right) \varepsilon_{j}\right) .
$$

Thus, $v(x)=w(x)$ for every $x \in \mathbb{R}^{2}$.

Clearly, $\Delta w(x)=V(x) w(x)$, because $w$ is $L$-harmonic. Since $w$ has logarithmic growth (see (1.9)), there is $C_{1}>0$ such that

$$
w(x)=(2 \pi)^{-1} \int_{\mathbb{R}^{2}} V(y) w(y) \ln |x-y| d y+C_{1} .
$$

It follows from (3.6) that

$$
|\nabla w(x)| \leq C(1+|x|)^{-1}
$$


and

$$
\left|w(x)-w\left(x^{\prime}\right)\right| \leq C \quad \text { provided } \quad|x| / 2 \leq\left|x^{\prime}\right| \leq 2|x| .
$$

Another direct consequence of (3.6) is the following lemma:

Lemma 3.4. Let a be a classical $H^{1}\left(\mathbb{R}^{2}\right)$-atom. Set $g=\left(I-V \Delta^{-1}\right) a$. Then

$$
\int g(x) w(x) d x=0 .
$$

\section{Atoms}

We start this section by proving the following lemma:

Lemma 4.1. Let $\varepsilon_{j}$ be a sequence as in the definition of the function $w(x)$. Let $b$ be an atom for $H_{w}^{1}$, at. Then

$$
\lim _{j \rightarrow \infty}\left(V \Delta_{\varepsilon_{j}}^{-1}\left(V L^{-1}-V L_{\varepsilon_{j}}^{-1}\right)\right) b(x)=0
$$

uniformly in $x$.

Proof. Since $\operatorname{supp} V \subset B(0,1)$, it is enough to consider $|x| \leq 1$. Let $U_{\varepsilon}(x, y)$ denote the integral kernel of the operator $\left.-V \Delta_{\varepsilon}^{-1}\left(V L^{-1}-V L_{\varepsilon}^{-1}\right)\right)$. Fix an atom $b$ for $H_{w \text {, at }}^{1}($ see $(1.6)-(1.7))$. Let $r$ be such that $\operatorname{supp} b \subset B(0, r)$. Then for $|x| \leq 1$ and $|y| \leq r$ we have

$$
\begin{aligned}
U_{\varepsilon}(x, y)= & \int_{0}^{\infty} \int_{\mathbb{R}^{2}} \int_{0}^{\infty} V(x) P_{u}(x-z) e^{-\varepsilon u} V(z) K_{t}(z, y)\left(1-e^{-\varepsilon t}\right) d t d z d u \\
= & \int_{0}^{1} \int_{\mathbb{R}^{2}} \int_{0}^{3} \ldots d t d z d u+\int_{0}^{1} \int_{\mathbb{R}^{2}} \int_{3}^{\infty} \ldots d t d z d u \\
& +\int_{1}^{\infty} \int_{\mathbb{R}^{2}} \int_{0}^{\infty} \ldots d t d z d u \\
:= & I_{1}(x, y)+I_{2}(x, y)+I_{3}(x, y) .
\end{aligned}
$$

By the mean value theorem and (1.2) we obtain that

$$
\begin{aligned}
I_{1}(x, y) & \leq C V(x) \int_{0}^{1} \int_{\mathbb{R}^{2}} \int_{0}^{3} P_{u}(x-z) V(z)\left\|K_{t}\right\|_{\infty} \varepsilon t d t d z d u \\
& \leq C \varepsilon V(x)\|V\|_{\infty} .
\end{aligned}
$$

To estimate $I_{2}$ we apply (2.12) and obtain

$$
\begin{aligned}
I_{2}(x, y) & \leq C V(x) \int_{0}^{1} \int_{\mathbb{R}^{2}} \int_{3}^{\infty} P_{u}(x-z) V(z) \frac{\ln (2+r)}{t(\ln t)^{2}}\left(1-e^{-\varepsilon t}\right) d t d z d u \\
& \leq C V(x)\|V\|_{\infty} \int_{1}^{\infty} \frac{\ln (2+r)}{t(\ln t)^{2}}\left(1-e^{-\varepsilon t}\right) d t \\
& \leq C V(x) \ln (2+r)\|V\|_{\infty}\left(\ln \varepsilon^{-1}\right)^{-1} .
\end{aligned}
$$


In order to deal with $I_{3}$ we split the integral as follows:

$$
\begin{aligned}
& I_{3}(x, y) \\
& =\int_{1}^{\infty} \int_{\mathbb{R}^{2}} \int_{0}^{\infty} V(x)\left(P_{u}(x-z)-P_{u}(0)\right) e^{-\varepsilon u} V(z) K_{t}(z, y)\left(1-e^{-\varepsilon t}\right) d t d z d u \\
& \quad+\int_{1}^{\infty} \int_{\mathbb{R}^{2}} \int_{0}^{\infty} V(x) P_{u}(0) e^{-\varepsilon u} V(z) K_{t}(z, y)\left(1-e^{-\varepsilon t}\right) d t d z d u \\
& =I_{3}^{\prime}(x, y)+V(x) w_{\varepsilon}(y) .
\end{aligned}
$$

By the mean value theorem,

$$
\begin{aligned}
\left|I_{3}^{\prime}(x, y)\right| \leq & C \int_{1}^{\infty} \int_{\mathbb{R}^{2}} \int_{0}^{\infty} V(x) u^{-3 / 2} V(z) K_{t}(z, y)\left(1-e^{-\varepsilon t}\right) d t d z d u \\
\leq & C V(x) \int_{\mathbb{R}^{2}} \int_{0}^{1 / \sqrt{\varepsilon}} V(z) K_{t}(z, y) \varepsilon t d t d z \\
& +C V(x) \int_{\mathbb{R}^{2}} \int_{1 / \sqrt{\varepsilon}}^{\infty} V(z) K_{t}(z, y) d t d z \\
\leq & C V(x) \sqrt{\varepsilon}\|V\|_{L^{1}}+C V(x)\|V\|_{L^{1}}\left(\ln \varepsilon^{-1}\right)^{-1},
\end{aligned}
$$

where in the last inequality we have used (2.12). Hence we obtain the lemma from (4.2)-(4.6) and (1.7), because

$$
\lim _{j \rightarrow \infty} \int_{\mathbb{R}^{2}} V(x) w_{\varepsilon_{j}}(y) b(y) d y=V(x) \int_{\mathbb{R}^{2}} w(y) b(y) d y=0 .
$$

The goal of this section is to prove the following proposition, which will be used in the proof of the second inequality in (1.10).

Proposition 4.2. There is a constant $C>0$ such that if $b$ is an atom for the Hardy space $H_{w, \text { at }}^{1}$ associated with a ball $B(x, r)$, then there exist a finite sequence $a_{k}$ of classical $H^{1}\left(\mathbb{R}^{2}\right)$-atoms and a sequence $\lambda_{k} \in \mathbb{C}$ such that

$$
\text { each atom } a_{k} \text { is associated with a ball } B\left(x_{k}, r_{k}\right) \text { with } r_{m} \leq \max (1, r) \text {, }
$$

$$
\sum_{k}\left|\lambda_{k}\right| \leq C, \quad \text { and } \quad b=\sum_{k} \lambda_{k}\left(I-V \Delta^{-1}\right) a_{k} .
$$

The proof of the proposition consists of Lemmas 4.3, 4.7, 4.8, 4.9, and Corollary 4.6 below.

For $n, m \in \mathbb{N} \cup\{0\}, n<m$, let $R_{n, m}=\left\{x \in \mathbb{R}^{2}: 2^{n / 2} \leq|x| \leq 2^{m / 2}\right\}$. The following decomposition will be frequently used here:

$$
\left|R_{n, n+1}\right|^{-1} \chi_{R_{n, n+1}}=\left|R_{0,1}\right|^{-1} \chi_{R_{0,1}}+\sum_{k=1}^{n} f_{k},
$$


where

$$
f_{k}=\left|R_{k, k+1}\right|^{-1} \chi_{R_{k, k+1}}-\left|R_{k-1, k}\right|^{-1} \chi_{R_{k-1, k}} .
$$

Observe that for $k=1,2, \ldots, n$ we have

$$
\begin{aligned}
& \operatorname{supp} f_{k} \subset R_{k-1, k+1}, \quad \int_{\mathbb{R}^{2}} f_{k}(y) d y=0 \\
& \left\|f_{k}\right\|_{\infty} \leq \frac{C}{\left|R_{k-1, k+1}\right|}, \quad\left\|f_{k}\right\|_{H^{1}\left(\mathbb{R}^{2}\right)} \leq C,
\end{aligned}
$$

with a constant $C$ independent of $n$ and $k$. Consequently,

$$
\begin{gathered}
\left|R_{n, n+1}\right|^{-1} \chi_{R_{n, n+1}}=\left|R_{0,1}\right|^{-1} \chi_{R_{0,1}}+\sum_{k=1}^{n} V \Delta^{-1} f_{k}+\sum_{k=1}^{n}\left(I-V \Delta^{-1}\right) f_{k} \\
\left\|\left|R_{0,1}\right|^{-1} \chi_{R_{0,1}}+\sum_{k=1}^{n} V \Delta^{-1} f_{k}\right\|_{\infty} \leq C(n+1) .
\end{gathered}
$$

Lemma 4.3. Assume that $b$ is an $H_{w}^{1}$ at -atom such that $\operatorname{supp} b \subset B(0,3)$ and $\|b\|_{\infty} \leq|B(0,3)|^{-1}$. Then there is an $H^{1}\left(\mathbb{R}^{2}\right)$-atom a such that $\operatorname{supp} a \subset B(0,3)$, $\|a\|_{\infty} \leq C$, and $b=\left(I-V \Delta^{-1}\right) a$.

Proof. Set $a=\left(I-V L^{-1}\right) b$. Clearly, supp $a \subset B(0,3),\|a\|_{\infty} \leq C$ and, by Lemma 2.4, $\int a=0$. Moreover, using (2.14) combined with (2.15), and Lemmas 2.5 and 4.1, we get $b=\left(I-V \Delta^{-1}\right) a$.

Lemma 4.4. Let $b$ be a function such that $\operatorname{supp} b \subset R_{n, n+1},\|b\|_{\infty} \leq 2^{-n}$, and $\int b w=0$. Then there exists a finite sequence $\left\{a_{j}\right\}_{j=0}^{n+1}$ of classical $H^{1}\left(\mathbb{R}^{2}\right)$-atoms and a sequence $\left\{\lambda_{j}\right\}_{j=0}^{n+1}$ of complex numbers such that

$$
b=\sum_{j=0}^{n+1} \lambda_{j}\left(I-V \Delta^{-1}\right) a_{j}, \quad \sum_{j=0}^{n+1}\left|\lambda_{j}\right| \leq C .
$$

The constant $C$ is independent of $b$.

Proof. Set

$$
\kappa=\int_{\mathbb{R}^{2}} b(x) d x
$$

Fix $x_{0} \in R_{n, n+1}$. By the assumptions on $b$ and (3.8), we obtain

$$
|\kappa|=w\left(x_{0}\right)^{-1}\left|\int_{\mathbb{R}^{2}} b(x)\left(w\left(x_{0}\right)-w(x)\right) d x\right| \leq C w\left(x_{0}\right)^{-1} \leq C n^{-1},
$$

where in the last inequality we have used (1.9). Applying (4.9) (see also (4.11)), 
we have

$$
\begin{aligned}
b= & \frac{\kappa}{\left|R_{n, n+1}\right|} \chi_{R_{n, n+1}}+\left(b-\frac{\kappa}{\left|R_{n, n+1}\right|} \chi_{R_{n, n+1}}\right) \\
= & \kappa\left|R_{0,1}\right|^{-1} \chi_{R_{0,1}}+\kappa \sum_{k=1}^{n} V \Delta^{-1} f_{k}+V \Delta^{-1}\left(b-\frac{\kappa}{\left|R_{n, n+1}\right|} \chi_{R_{n, n+1}}\right) \\
& +\left(I-V \Delta^{-1}\right)\left(b-\frac{\kappa}{\left|R_{n, n+1}\right|} \chi_{R_{n, n+1}}\right)+\kappa \sum_{k=1}^{n}\left(I-V \Delta^{-1}\right) f_{k} .
\end{aligned}
$$

Clearly, $b-\kappa\left|R_{n, n+1}\right|^{-1} \chi_{R_{n, n+1}}$ and $f_{k}, k=1,2, \ldots, n$, are multiples of $H^{1}\left(\mathbb{R}^{2}\right)$ atoms with a universal constant factor independent of $b$ (see (4.10) and (4.13)). Moreover, the function

$$
g(x)=\kappa\left|R_{0,1}\right|^{-1} \chi_{R_{0,1}}+\kappa \sum_{k=1}^{n} V \Delta^{-1} f_{k}+V \Delta^{-1}\left(b-\frac{\kappa}{\left|R_{n, n+1}\right|} \chi_{R_{n, n+1}}\right)
$$

is supported in $B(0, \sqrt{2})$, and, thanks to (4.12) and (4.13), we have that $\|g\|_{\infty} \leq C$. Since $\int b w=0$, we conclude from (4.14) and Lemma 3.4 that $\int g w=0$. Therefore, by Lemma $4.3, g=\left(I-V \Delta^{-1}\right) f_{0}$, where $f_{0}$ is a multiple of a classical $H^{1}\left(\mathbb{R}^{2}\right)$ atom. Hence, Lemma 4.4 follows form (4.14), (4.12), and (4.13).

Lemma 4.5. Assume that $b$ is an $H_{w \text {, at }}^{1}$-atom associated with $B\left(0,2^{n / 2}\right), n>1$. Then there is a sequence of functions $b_{k}, k=0,1, \ldots, n-1$, such that

$$
\begin{aligned}
& \left\|b_{k}\right\|_{\infty} \leq C 2^{-n}, \quad \int b_{k} w=0, \\
& \operatorname{supp} b_{0} \subset B(0, \sqrt{2}), \quad \operatorname{supp} b_{k} \subset R_{k-1, k+1} \quad \text { for } k=1,2, \ldots, n-1, \\
& b=\sum_{k=0}^{n-1} b_{k} .
\end{aligned}
$$

Proof. Let $B_{0}=B(0,1)$ and $A=\left|B\left(0,2^{n / 2}\right)\right|^{-1}=\pi^{-1} 2^{-n}$. By the assumptions, $\|b\|_{\infty} \leq A$. Define

$$
s_{0}=1+\frac{w(B(0,1))}{w\left(R_{0,1}\right)}, \quad s_{k}=1+s_{k-1} \frac{w\left(R_{k-1, k}\right)}{w\left(R_{k, k+1}\right)} \quad \text { for } k=1,2, \ldots
$$

where $w(U)=\int_{U} w(x) d x$ for a measurable set $U \subset \mathbb{R}^{2}$. Formula (3.6) implies that

$$
0<w\left(R_{k-1, k}\right) / w\left(R_{k, k+1}\right) \leq r<1 \quad \text { for } k \geq 2 .
$$

Hence the sequence $s_{k}$ is bounded. Set

$$
b=\left(b \chi_{B_{0}}-c_{0} \chi_{R_{0,1}}\right)+\left(c_{0} \chi_{R_{0,1}}+b \chi_{R_{0, n}}\right):=b_{0}+\widetilde{b}_{0},
$$

where

$$
c_{0}=\frac{1}{w\left(R_{0,1}\right)} \int_{B_{0}} b w
$$


Obviously, $\left|c_{0}\right| \leq A w\left(B_{0}\right) w\left(R_{0,1}\right)^{-1}$. We easily see that

$$
\begin{gathered}
\int b_{0} w=\int \widetilde{b}_{0} w=0, \\
\operatorname{supp} b_{0}=B(0, \sqrt{2}), \quad \operatorname{supp} \widetilde{b}_{0} \subset R_{0, n}, \\
\left|b_{0}(x)\right| \leq A s_{0}, \\
\left|\widetilde{b}_{0}(x)\right| \leq \begin{cases}A s_{0} & \text { for } x \in R_{0,1}, \\
A & \text { for } x \in R_{1, n} .\end{cases}
\end{gathered}
$$

Now we decompose $\widetilde{b}_{0}$ :

$$
\widetilde{b}_{0}=\left(\widetilde{b}_{0} \chi_{R_{0,1}}-c_{1} \chi_{R_{1,2}}\right)+\left(c_{1} \chi_{R_{1,2}}+b \chi_{R_{1, n}}\right):=b_{1}+\widetilde{b}_{1},
$$

where $c_{1}=w\left(R_{1,2}\right)^{-1} \int_{R_{0,1}} \widetilde{b}_{0} w$. Clearly, $\int b_{1} w=\int \widetilde{b}_{1} w=0$. Moreover,

$$
\left|c_{1}\right| \leq A s_{0} \frac{w\left(R_{0,1}\right)}{w\left(R_{1,2}\right)}
$$

$\operatorname{supp} b_{1} \subset R_{0,2}, \operatorname{supp} \widetilde{b}_{1} \subset R_{1, n}$, and

$$
\begin{gathered}
\left|b_{1}(x)\right| \leq \begin{cases}A s_{0} & \text { for } x \in R_{0,1}, \\
A s_{0} \frac{w\left(R_{0,1}\right)}{w\left(R_{1,2}\right)} & \text { for } x \in R_{1,2},\end{cases} \\
\left|\widetilde{b}_{1}(x)\right| \leq \begin{cases}A s_{1} & \text { for } x \in R_{1,2}, \\
A & \text { for } x \in R_{2, n}\end{cases}
\end{gathered}
$$

Then we decompose $\widetilde{b}_{1}$ :

$$
\widetilde{b}_{1}=\left(\widetilde{b}_{1} \chi_{R_{1,2}}-c_{2} \chi_{R_{2,3}}\right)+\left(c_{2} \chi_{R_{2,3}}+b \chi_{R_{2, n}}\right):=b_{2}+\widetilde{b}_{2},
$$

where $c_{2}=w\left(R_{2,3}\right)^{-1} \int_{R_{1,2}} \widetilde{b}_{1} w$. We have $\int b_{2} w=\int \widetilde{b}_{2} w=0$, and

$$
\left|c_{2}\right| \leq A s_{1} \frac{w\left(R_{1,2}\right)}{w\left(R_{2,3}\right)} .
$$

In addition, supp $b_{2} \subset R_{1,3}, \operatorname{supp} \widetilde{b}_{2} \subset R_{2, n}$, and

$$
\begin{aligned}
& \left|b_{2}(x)\right| \leq \begin{cases}A s_{1} & \text { for } x \in R_{1,2}, \\
A s_{1} \frac{w\left(R_{1,2}\right)}{w\left(R_{2,3}\right)} & \text { for } x \in R_{2,3},\end{cases} \\
& \left|\widetilde{b}_{2}(x)\right| \leq \begin{cases}A s_{2} & \text { for } x \in R_{2,3}, \\
A & \text { for } x \in R_{3, n} .\end{cases}
\end{aligned}
$$


We continue the procedure and get functions $b_{k}$ and $\widetilde{b}_{k}, k=1,2, \ldots, n-2$, such that $\operatorname{supp} b_{k} \subset R_{k-1, k+1}, \operatorname{supp} \widetilde{b}_{k} \subset R_{k, n}, \int b_{k} w=\int \widetilde{b}_{k} w=0, b=\widetilde{\vec{b}}_{n-2}+\sum_{k=0}^{n-2} b_{k}$ and

$$
\begin{aligned}
& \left|b_{k}(x)\right| \leq \begin{cases}A s_{k-1} & \text { for } x \in R_{k-1, k}, \\
A s_{k-1} \frac{w\left(R_{k-1, k}\right)}{w\left(R_{k, k+1}\right)} & \text { for } x \in R_{k, k+1},\end{cases} \\
& \left|\widetilde{b}_{k}(x)\right| \leq \begin{cases}A s_{k} & \text { for } x \in R_{k, k+1}, \\
A & \text { for } x \in R_{k+1, n} .\end{cases}
\end{aligned}
$$

Set $b_{n-1}=\widetilde{b}_{n-2}$. From the above we see that $\left|b_{k}\right| \leq C 2^{-n}$, with $C$ independent of $b$.

As a direct consequence of Lemmas 4.4 and 4.5, we obtain the following corollary:

Corollary 4.6. The conclusion of Proposition 4.2 holds for every $H_{w}^{1}$, at-atom $b$ associated with a ball $B\left(0,2^{n / 2}\right), n>1$.

Lemma 4.7. The conclusion of Proposition 4.2 holds for every $H_{w}^{1}$, at-atom $b$ associated with a ball $B\left(x_{0}, r\right),\left|x_{0}\right|<2, r<1$.

Proof. Set $c_{0}=\left|B\left(x_{0}, r\right)\right|^{-1} \int b(x) d x$. By (1.6)-(1.7) and the mean value theorem,

$$
\left|c_{0}\right|=\left|B\left(x_{0}, r\right)\right|^{-1} w\left(x_{0}\right)^{-1}\left|\int_{\mathbb{R}^{2}} b(x)\left(w\left(x_{0}\right)-w(x)\right) d x\right| \leq C r^{-1} .
$$

Define $B_{j}=B\left(x_{0}, 2^{j} r\right), c_{j}=c_{0} \frac{\left|B_{0}\right|}{\left|B_{j}\right|}, j=0,1, \ldots,\left[\log _{2} r^{-1}\right]=k$. Then, $b=\left(b-c_{0} \chi_{B_{0}}\right)+c_{0}\left|B_{0}\right| \sum_{j=0}^{k}\left(\left|B_{j}\right|^{-1} \chi_{B_{j}}-\left|B_{j+1}\right|^{-1} \chi_{B_{j+1}}\right)+c_{0}\left|B_{0}\right|\left|B_{k+1}\right|^{-1} \chi_{B_{k+1}}$.

Set $a_{0}=\left(b-c_{0} \chi_{B_{0}}\right)$ and $a_{j+1}=\left|B_{j}\right|^{-1} \chi_{B_{j}}-\left|B_{j+1}\right|^{-1} \chi_{B_{j+1}}$, for $j=0,1, \ldots, k$. Clearly $a_{j}, j=0,1, \ldots, k$, are multiples of classical $H^{1}\left(\mathbb{R}^{2}\right)$-atoms with a constant factor independent of $b$. So,

$$
\begin{aligned}
b= & \left(I-V \Delta^{-1}\right) a_{0}+\left(I-V \Delta^{-1}\right) \sum_{j=1}^{k} c_{0}\left|B_{0}\right| a_{j} \\
& +V \Delta^{-1} a_{0}+V \Delta^{-1} \sum_{j=1}^{k} c_{0}\left|B_{0}\right| a_{j}+c_{0} \frac{\left|B_{0}\right|}{\left|B_{k+1}\right|} \chi_{B_{k+1}} .
\end{aligned}
$$

Note that $\sum_{j=1}^{k} c_{0}\left|B_{0}\right| \leq C$. Further, from Lemma 3.4 we deduce that

$$
V \Delta^{-1}\left(a_{0}+\sum_{j=1}^{k} c_{0}\left|B_{0}\right| a_{j}\right)+c_{0} \frac{\left|B_{0}\right|}{\left|B_{k+1}\right|} \chi_{B_{k+1}}
$$

is a multiple of an $H_{w \text {, at }}^{1}$-atom associated with the ball $B(0,3)$. Hence, the lemma follows by applying (4.16) and Lemma 4.3. 
Lemma 4.8. Assume that $b$ is an $H_{w \text {, at }}^{1}$-atom associated with $B\left(x_{0}, r\right),\left|x_{0}\right|>1$, $r \leq 1$. Then the conclusion of Proposition 4.2 about the decomposition holds.

Proof. Let $n=\left[\log _{2} r^{-1}\right], s=\left[\left|x_{0}\right|\right], B_{k}=B\left(x_{0}, 2^{k} r\right)$, for $k=0,1, \ldots, n$, and $B_{n+j}=B\left(\frac{s-j}{s} x_{0}, 2^{n} r\right)$, for $j=1, \ldots, s$. Put $c_{0}=\left|B_{0}\right|^{-1} \int b(x) d x$,

$$
\begin{aligned}
& a_{0}=b-c_{0} \chi_{B_{0}}, \quad \lambda_{0}=1 ; \\
& a_{k}=\left|B_{k-1}\right|^{-1} \chi_{B_{k-1}}-\left|B_{k}\right|^{-1} \chi_{B_{k}}, \quad \lambda_{k}=c_{0}\left|B_{0}\right|, \quad k=1,2, \ldots, n ; \\
& a_{n+j}=\left|B_{n}\right|^{-1}\left(\chi_{B_{n+j-1}}-\chi_{B_{n+j}}\right), \quad \lambda_{n+j}=c_{0}\left|B_{0}\right|, \quad j=1,2, \ldots, s .
\end{aligned}
$$

Then, from (1.6), (1.7), (3.7), and the mean value theorem we obtain that

$$
\left|c_{0}\right| w\left(x_{0}\right)\left|B_{0}\right|\left|x_{0}\right| \leq C r .
$$

Moreover, the $a_{m}$ are multiples of classical $H^{1}\left(\mathbb{R}^{2}\right)$-atoms (with a constant factor independent of $b$ ) and

$$
b=\sum_{m=0}^{n+s} \lambda_{m} a_{m}+c_{0} \frac{\left|B_{0}\right|}{\left|B_{n}\right|} \chi_{B_{n+s}} .
$$

Clearly, $\sum_{m=0}^{n+s}\left|\lambda_{m}\right| \leq C$, and

$$
b=\sum_{m=0}^{n+s} \lambda_{m}\left(I-V \Delta^{-1}\right) a_{m}+\sum_{m=0}^{n+s} \lambda_{m} V \Delta^{-1} a_{m}+c_{0} \frac{\left|B_{0}\right|}{\left|B_{n+s}\right|} \chi_{B_{n+s}} .
$$

The function $g=\sum_{m=0}^{n+s} \lambda_{m} V \Delta^{-1} a_{m}+c_{0}\left|B_{0}\right|\left|B_{n+s+1}\right|^{-1} \chi_{B_{n+s+1}}$ is supported in $B(0,2),\|g\|_{\infty} \leq C$, and, by Lemma 3.4, $\int g(x) w(x) d x=0$. The proof is completed by the use of (4.17) and Lemma 4.3.

Lemma 4.9. The conclusion of Proposition 4.2 holds for every $H_{w, \text { at }}^{1}$-atom $b$ associated with a ball $B\left(x_{0}, r\right),\left|x_{0}\right|>2 r>2$.

Proof. Let $s=\left[\left|x_{0}\right| / r\right], n=\left[\log _{2} r\right]+1, B_{k}=B\left(\frac{s-k}{s} x_{0}, r\right), k=0,1, \ldots, s$, and $B_{s+j}=B\left(0,2^{-j} r\right), j=1,2, \ldots, n$. Set $c_{0}=\left|B_{0}\right|^{-1} \int^{s} b(x) d x$. We have

$$
\left|c_{0}\right| w\left(x_{0}\right)\left|B_{0}\right|\left|x_{0}\right| \leq r .
$$

Define

$$
\begin{aligned}
a_{0} & =b-c_{0} \chi_{B_{0}}, \lambda_{0}=1 ; \\
a_{k} & =\left|B_{k}\right|^{-1}\left(\chi_{B_{k-1}}-\chi_{B_{k}}\right), \quad \lambda_{k}=c_{0}\left|B_{0}\right|, k=1, \ldots, s ; \\
a_{s+j} & =\left|B_{s+j-1}\right|^{-1} \chi_{B_{s+j-1}}-\left|B_{s+j}\right|^{-1} \chi_{B_{s+j}}, \quad \lambda_{s+j}=c_{0}\left|B_{s}\right|, j=1,2, \ldots, n .
\end{aligned}
$$

The functions $a_{k}, k=0,1, \ldots, s+n$, are multiples of the classical $H^{1}\left(\mathbb{R}^{2}\right)$-atoms. By the same arguments as in the proof of the previous lemma,

$$
b=\sum_{m=0}^{s+n}\left(I-V \Delta^{-1}\right) \lambda_{m} a_{m}+\sum_{m=0}^{s+n} V \Delta^{-1} \lambda_{m} a_{m}+c_{0} \frac{\left|B_{s}\right|}{\left|B_{s+n}\right|} \chi_{B_{s+n}}
$$


with $\sum_{m=0}^{s+n}\left|\lambda_{m}\right| \leq C$, the function

$$
g=\sum_{m=0}^{s+n} V \Delta^{-1} \lambda_{m} a_{m}+c_{0}\left|B_{s}\right|\left|B_{s+n}\right|^{-1} \chi_{B_{s+n}}
$$

being a multiple of an $H_{w \text {, at }}^{1}$-atom supported in $B(0,2)$. From equation (4.18) and Lemma 4.3, we get the decomposition.

\section{Boundedness of maximal functions}

The main goal of this section is to prove the second inequality in (1.10) of Theorem 1.1, that is, there exists a constant $C>0$ such that

$$
\left\|\mathcal{M}_{L} f\right\|_{L^{1}\left(\mathbb{R}^{2}\right)} \leq C\|f\|_{H_{w, \text { at }}^{1}} .
$$

Let us first note that if $a$ is a classical $H^{1}\left(\mathbb{R}^{2}\right)$-atom associated with a ball of radius $r>0$, then

$$
\left|P_{s} a(x)\right| \leq \begin{cases}C s^{-3 / 2} r & \text { for } s>r^{2} \\ C r^{-2} & \text { for } s \leq r^{2}\end{cases}
$$

Hence, there is $C>0$ such that

$$
\int_{0}^{\infty}\left|P_{s} a(x)\right| d s \leq C
$$

for every classical $H^{1}\left(\mathbb{R}^{2}\right)$-atom $a$.

The perturbation formula asserts that

$$
K_{t}=P_{t}-\int_{0}^{t}\left(K_{t-s}-K_{t}\right) V P_{s} d s-K_{t} \int_{0}^{t} V P_{s} d s .
$$

Let $f \in H^{1}\left(\mathbb{R}^{2}\right)$. Then

$$
\begin{aligned}
K_{t}\left(I-V \Delta^{-1}\right) f & =P_{t} f+K_{t} \int_{t}^{\infty} V P_{s} f d s+\int_{0}^{t}\left(K_{t}-K_{t-s}\right) V P_{s} f d s \\
& =P_{t} f+Q_{t} f+R_{t} f+S_{t} f,
\end{aligned}
$$

where

$$
\begin{aligned}
Q_{t} & =K_{t} \int_{t}^{\infty} V P_{s} d s \\
R_{t} & =\int_{0}^{t / 2}\left(K_{t}-K_{t-s}\right) V P_{s} d s \\
S_{t} & =\int_{t / 2}^{t}\left(K_{t}-K_{t-s}\right) V P_{s} d s .
\end{aligned}
$$


Lemma 5.1. There exists a constant $C>0$ such that, for every $H^{1}\left(\mathbb{R}^{2}\right)$-atom $a$, one has

$$
\left\|\sup _{0<t \leq 4}\left|Q_{t} a\right|\right\|_{L^{1}} \leq C, \quad\left\|\sup _{0<t \leq 4}\left|R_{t} a\right|\right\|_{L^{1}} \leq C, \quad\left\|\sup _{0<t \leq 4}\left|S_{t} a\right|\right\|_{L^{1}} \leq C .
$$

Proof. Let $a$ be a classical $H^{1}\left(\mathbb{R}^{2}\right)$-atom associated with a ball $B\left(x_{0}, r\right)$. Denote

$$
\mathbf{M}_{0} f(x)=\sup _{0<t \leq 4} P_{t}|f|(x) .
$$

Clearly, $\sup _{0<t \leq 4} K_{t}|f|(x) \leq \mathbf{M}_{0} f(x)$. Using (5.3), we get

$$
\sup _{0<t \leq 4}\left|Q_{t} a(x)\right| \leq C \mathbf{M}_{0} \chi_{B(0,1)}(x) .
$$

Similarly, for $0<t \leq 4$, we have

$$
\begin{aligned}
\left|R_{t} a(x)\right| & \leq K_{t}\left(\int_{0}^{t / 2} V\left|P_{s} a\right| d s\right)(x)+\int_{0}^{t / 2} K_{t-s} V\left|P_{s} a\right|(x) d s \\
& \leq C \mathbf{M}_{0} \chi_{B(0,1)}(x)+C \int_{0}^{t / 2} K_{t / 2} V\left|P_{s} a\right|(x) d s \\
& \leq C^{\prime} \mathbf{M}_{0} \chi_{B(0,1)}(x) .
\end{aligned}
$$

To deal with $S_{t}$ we apply (5.2) and obtain

$$
\begin{aligned}
\left|\int_{t / 2}^{t} K_{t-s} V P_{s} a(x) d s\right| & \leq \begin{cases}C \int_{t / 2}^{t}\left(K_{t-s} V\right)(x) r^{-2} d s & \text { if } t<r^{2} \\
C \int_{t / 2}^{t}\left(K_{t-s} V\right)(x) s^{-3 / 2} r d s & \text { if } t \geq r^{2}\end{cases} \\
& \leq C \mathbf{M}_{0} \chi_{B(0,1)}(x) .
\end{aligned}
$$

Similarly to (5.8), by the use of (5.3), we get

$$
\left|\int_{t / 2}^{t} K_{t} V P_{s} a(x) d s\right| \leq C \mathbf{M}_{0} \chi_{B(0,1)}(x) .
$$

Now the lemma follows from (5.7)-(5.10) and from the fact that $\mathbf{M}_{0}$ maps $\chi_{B(0,1)}$ into $L^{1}$.

Lemma 5.2. There exists a constant $C>0$ such that, for every classical $H^{1}\left(\mathbb{R}^{2}\right)$ atom a associated with a ball $B\left(x_{0}, r\right)$, one has

$$
\begin{aligned}
& \left\|\sup _{t>\max \left(r^{2}, 4\right)}\left|Q_{t} a\right|\right\|_{L^{1}} \leq C, \\
& \left\|\sup _{t>\max \left(r^{2}, 4\right)}\left|R_{t} a\right|\right\|_{L^{1}} \leq C, \\
& \left\|\sup _{t>\max \left(r^{2}, 4\right)}\left|S_{t} a\right|\right\|_{L^{1}} \leq C .
\end{aligned}
$$


Proof. From (5.2), for $t>\max \left(r^{2}, 4\right)$, we have

$$
\left|Q_{t} a(x)\right| \leq C K_{t}\left(\int_{0}^{\infty} V r s^{-3 / 2} d s\right)(x) \leq C r t^{-1 / 2} K_{t} V(x) .
$$

In order to deal with $R_{t}$ we note that for $0<s<t / 2$ one has

$$
\left|K_{t}(x, z)-K_{t-s}(x, z)\right| \leq C \frac{s}{t^{2}} \exp \left(-c|x-z| / t^{1 / 2}\right)=\frac{s}{t} \phi_{t}(x-z) .
$$

Hence, by (5.2), we obtain

$$
\begin{aligned}
\left|R_{t} a(x)\right| \leq & \int_{0}^{r^{2} / 2} \int_{\mathbb{R}^{2}} \frac{s}{t} \phi_{t}(x-z) V(z)\left|P_{s} a(z)\right| d z d s \\
& +\int_{r^{2} / 2}^{t / 2} \int_{\mathbb{R}^{2}} \frac{s}{t} \phi_{t}(x-z) V(z)\left|P_{s} a(z)\right| d z d s \\
\leq & \int_{0}^{r^{2} / 2} \int_{\mathbb{R}^{2}} \frac{s}{t} \phi_{t}(x-z) V(z) r^{-2} d z d s \\
& +\int_{r^{2} / 2}^{t / 2} \int_{\mathbb{R}^{2}} \frac{s}{t} \phi_{t}(x-z) V(z) r s^{-3 / 2} d z d s \\
\leq & C r t^{-1 / 2} \phi_{t} * V(x)+C t^{-1 / 2} \phi_{t} * V(x) .
\end{aligned}
$$

We now turn to estimating $S_{t} a$. Applying (5.2) we get

$$
\begin{aligned}
\left|\int_{t / 2}^{t} K_{t-s} V P_{s} a(x)\right| & \leq C \int_{t / 2}^{t} \int_{\mathbb{R}^{2}} K_{t-s}(x, z) V(z) r s^{-3 / 2} d z d s \\
& \leq C \frac{r}{t^{3 / 2}} \int_{0}^{t / 2} \int_{\mathbb{R}^{2}} K_{s}(x, z) V(z) d z d s \\
& =C \frac{r}{t^{3 / 2}} \int_{0}^{2}\left(K_{s} V\right)(x) d s+C \frac{r}{t^{3 / 2}} \int_{2}^{t / 2}\left(K_{s} V\right)(x) d s \\
& \leq C t^{-1} \mathbf{M}_{0} V(x)+C \frac{r}{t^{3 / 2}} \int_{2}^{t / 2}\left(K_{s} V\right)(x) d s .
\end{aligned}
$$

Set

$$
S(x)=\sup _{t>\max \left(r^{2}, 4\right)} \frac{r}{t^{3 / 2}} \int_{2}^{t / 2}\left(K_{s} V\right)(x) d s .
$$

If $|x| \leq 2$, then we use (2.12) and get

$$
S(x) \leq C \sup _{t>\max \left(r^{2}, 4\right)} \frac{r}{t^{3 / 2}} \int_{2}^{t / 2} s^{-1}(\ln s)^{-2} d s \leq C r(1+r)^{-3} .
$$


If $2 \leq|x| \leq \max (r, 2)$, then

$$
\begin{aligned}
S(x) & \leq C \sup _{t>\max \left(r^{2}, 4\right)}\left(\frac{r}{t^{3 / 2}} \int_{2}^{|x|}\left(K_{s} V\right)(x) d s+\frac{r}{t^{3 / 2}} \int_{|x|}^{t / 2}\left(K_{s} V\right)(x) d s\right) \\
& \leq C \sup _{t>\max \left(r^{2}, 4\right)}\left(\frac{r}{t^{3 / 2}} \int_{2}^{|x|} s^{-1} e^{-c|x|^{2} / s} d s+\frac{r}{t^{3 / 2}} \int_{|x|}^{t / 2} \frac{\ln |x|}{s(\ln s)^{2}} d s\right) \\
& \leq C e^{-c|x|}+C \sup _{t>\max \left(r^{2}, 4\right)} \frac{r}{t^{3 / 2}} \ln |x|\left(\frac{1}{\ln |x|}-\frac{1}{\ln t}\right) \\
& \leq C e^{-c|x|}+C(1+r)^{-2} .
\end{aligned}
$$

Finally, if $|x|>\max (r, 2)$, then

$$
\begin{aligned}
S(x) \leq & C \sup _{|x|^{2}>t>\max \left(r^{2}, 4\right)} \frac{r}{t^{3 / 2}} \int_{2}^{t / 2} K_{s} V(x) d s+C \sup _{t>|x|^{2}} \frac{r}{t^{3 / 2}} \int_{2}^{t / 2} K_{s} V(x) d s \\
\leq & C \sup _{|x|^{2}>t>\max \left(r^{2}, 4\right)} C \frac{r}{t^{3 / 2}} \int_{2}^{t} \frac{s^{N-1}}{|x|^{2 N}} d s \\
& +C \sup _{t>|x|^{2}} \frac{r}{t^{3 / 2}} \int_{2}^{|x|} K_{s} V(x) d s+C \sup _{t>|x|^{2}} \frac{r}{t^{3 / 2}} \int_{|x|}^{t / 2} K_{s} V(x) d s \\
\leq & C \frac{r}{|x|^{3}}+C \sup _{t>|x|^{2}} \frac{r}{t^{3 / 2}} \int_{2}^{|x|} \frac{s^{N-1}}{|x|^{2 N}} d s+C \sup _{t>|x|^{2}} \frac{r}{t^{3 / 2}} \int_{|x|}^{t / 2} \frac{\ln |x|}{s(\ln s)^{2}} d s \\
\leq & C \frac{r}{|x|^{3}} .
\end{aligned}
$$

The estimates for $\sup _{t>\max \left(r^{2}, 4\right)}\left|\int_{t / 2}^{t} K_{t} V P_{s} a d s\right|$ are similar to those we have provided for $Q_{t}$ (see (5.12)). Observe that

$$
\left\|\sup _{t>\max \left(r^{2}, 4\right)} r t^{-1 / 2} \phi_{t} * V(x)\right\|_{L^{1}\left(\mathbb{R}^{2}\right)} \leq C .
$$

Hence, taking together (5.12)-(5.18) we obtain the lemma.

Proof of (5.1). It suffices to prove that $\left\|\mathcal{M}_{L} b\right\|_{L^{1}} \leq C$ for $b$ being any $H_{w \text {, at }}^{1}$-atom. If an atom $b$ is associated with a ball $B\left(x_{0}, r\right)$, then, from (1.2) one easily concludes that $\left\|\sup _{0<t \leq r^{2}}\left|K_{t} b\right|\right\|_{L^{1}} \leq C$. Thus there remains only the case $t>r^{2}$. To this end, we note that by Proposition 4.2 it is enough to prove that $\| \sup _{t>r^{2}} \mid K_{t}(I-$ $\left.V \Delta^{-1}\right) a \mid \|_{L^{1}} \leq C$ for every classical $H^{1}\left(\mathbb{R}^{2}\right)$-atom $a$. This is a direct consequence of (5.5), and Lemmas 5.1 and 5.2.

\section{Atomic decomposition}

The goal of this section is to prove the first inequality in (1.10) of Theorem 1.1,

$$
\|f\|_{H_{w, \text { at }}^{1}} \leq C\left\|\mathcal{M}_{L} f\right\|_{L^{1}},
$$

with a constant $C>0$ independent of $f$. For this purpose we shall use results about Hardy spaces associated with Schrödinger operators proved in [15]. 
Let $\left\{T_{t}\right\}_{t>0}$ be a semigroup of linear operators generated by a Schrödinger operator $\mathcal{L}=\Delta-\mathcal{V}$ on $\mathbb{R}^{d}$, where $\mathcal{V}$ is a nonnegative locally integrable potential. Let $M \geq 1$ be a positive integer. A function $\widetilde{a} \in L^{1}\left(\mathbb{R}^{d}\right)$ is called a $(1,2, M)$-atom associated with $\mathcal{L}$ (see Section 2 of [15]) if there exists a function $\widetilde{b}$ which belongs to the domain of the operator $\mathcal{L}^{M}$ considered on $L^{2}\left(\mathbb{R}^{d}\right)$ and a ball $B=B\left(x_{0}, r_{B}\right)$ such that

(i) $\widetilde{a}=L^{M} \widetilde{b}$;

(ii) $\operatorname{supp} \mathcal{L}^{k} \widetilde{b} \subset B, k=0,1, \ldots, M$;

(iii) $\left\|\mathcal{L}^{k} \widetilde{b}\right\|_{L^{2}\left(\mathbb{R}^{d}\right)} \leq r_{B}^{2 M-2 k}|B|^{-1 / 2}, k=0,1, \ldots, M$.

The atomic norm $\|f\|_{H_{\mathcal{L}, \text { at, }}^{1}}$ is defined by

$$
\|f\|_{H_{\mathcal{L}, a t, M}^{1}}=\inf \left\{\sum_{j=1}^{\infty}\left|\lambda_{j}\right|\right\}
$$

where the infimum is taken over all representations $f=\sum_{j=1}^{\infty} \lambda_{j} \widetilde{a}_{j}$, where $\lambda_{j} \in \mathbb{C}$ and the $a_{j}$ are $(1,2, M)$-atoms. The following atomic decomposition was shown in Theorem 8.2 of [15]. For every integer $M \geq 1$ there is a constant $C_{M}>0$ which depends on $M$ and $d$ such that

$$
C_{M}^{-1}\|f\|_{H_{\mathcal{L}, a t, M}^{1}} \leq\left\|\mathcal{M}_{\mathcal{L}} f\right\|_{L^{1}\left(\mathbb{R}^{d}\right)} \leq C_{M}\|f\|_{H_{\mathcal{L}, a t, M}^{1}} .
$$

Using (6.2) we shall prove another atomic decomposition for elements of the Hardy space $H^{1}$ associated with $\mathcal{L}$. We say that a function a is a generalized $(1, q)$ atom $(1<q \leq \infty)$ for $\mathbf{H}_{q, \mathcal{L}, \text { at }}^{1}$ if there exists a function $\mathbf{b}$ and a ball $B=B\left(x_{0}, r_{B}\right)$ such that

(i) $\operatorname{supp} \mathbf{b} \subset B$;

(ii) $\|\mathbf{b}\|_{L^{q\left(\mathbb{R}^{d}\right)}} \leq|B|^{1 / q-1}$;

(iii) $\mathbf{a}=\left(I-T_{r_{B}^{2}}\right) \mathbf{b}$.

The norm $\|f\|_{\mathbf{H}_{q, \mathcal{L}, \text { at }}^{1}}$ is defined by

$$
\|f\|_{\mathbf{H}_{q, \mathcal{L}, \mathbf{a t}}^{1}}=\inf \left\{\sum_{j}\left|\lambda_{j}\right|: f=\sum_{j} \lambda_{j} \mathbf{a}_{j}\right\}
$$

where the infimum is taken over all decompositions $f=\sum_{j} \lambda_{j} \mathbf{a}_{j}, \lambda_{j} \in \mathbb{C}$, and the $\mathbf{a}_{j}$ are generalized $(1, q)$-atoms.

Proposition 6.1. There is a constant $C_{q}$ such that

$$
\left\|\mathcal{M}_{\mathcal{L}} \mathbf{a}\right\|_{L^{1}\left(\mathbb{R}^{d}\right)} \leq C_{q} .
$$

for every generalized $(1, q)$-atom $\mathbf{a}$. 
Proof. Using standard arguments (see, e.g., [13]) we have that there is a constant $C_{q}$ such that

$$
\left\|\sup _{0<t<r_{B}^{2}}\left|T_{t} \mathbf{a}(x)\right|\right\|_{L^{1}(d x)} \leq 2\left\|\sup _{0<t<2 r_{B}^{2}} \mid T_{t} \mathbf{b}(x)\right\|_{L^{1}(d x)} \leq C_{q}
$$

for every generalized $(1, q)$-atom a.

We now consider $\sup _{t>r_{B}^{2}}\left|K_{t} \mathbf{a}(x)\right|$. Using the functional calculus for Schrödinger operators, see e.g. [14], one gets $\left\|\mathcal{L}^{2} T_{t}\right\|_{L^{1}\left(\mathbb{R}^{d}\right) \rightarrow L^{1}\left(\mathbb{R}^{d}\right)} \leq C t^{-2}$. Hence,

$$
\begin{aligned}
\sup _{t \geq r_{B}^{2}}\left|T_{t} \mathbf{a}(x)\right| & =\sup _{t \geq r_{B}^{2}}\left|\left(T_{t+r_{B}^{2}}-T_{t}\right) \mathbf{b}(x)\right|=\sup _{t \geq r_{B}^{2}}\left|\int_{t}^{t+r_{B}^{2}} \mathcal{L} K_{s} \mathbf{b}(x) d s\right| \\
& =\sup _{t \geq r_{B}^{2}}\left|\int_{t}^{t+r_{B}^{2}} \int_{s}^{\infty} \mathcal{L}^{2} K_{u} \mathbf{b}(x) d u d s\right| \\
& \leq \sup _{t \geq r_{B}^{2}} \int_{t}^{t+r_{B}^{2}} \int_{r_{B}^{2}}^{\infty}\left|\mathcal{L}^{2} K_{u} \mathbf{b}(x)\right| d u d s=r_{B}^{2} \int_{r_{B}^{2}}^{\infty}\left|\mathcal{L}^{2} K_{u} \mathbf{b}(x)\right| d u
\end{aligned}
$$

and, consequently,

$$
\left\|\sup _{t \geq r_{B}^{2}}\left|T_{t} \mathbf{a}(x)\right|\right\|_{L^{1}(d x)} \leq C
$$

Lemma 6.2. There is a constant $C>0$ such that

$$
\|f\|_{\mathbf{H}_{\infty, \mathcal{L}, \text { at }}^{1}} \leq C\left\|\mathcal{M}_{\mathcal{L}} f\right\|_{L^{1}\left(\mathbb{R}^{d}\right)} .
$$

Proof. Assume that $\left\|\mathcal{M}_{\mathcal{L}} f\right\|_{L^{1}\left(\mathbb{R}^{d}\right)}<\infty$. Then, by virtue of $(6.2)$,

$$
f=\sum_{j} \lambda_{j} \widetilde{a}_{j}, \quad \sum_{j}\left|\lambda_{j}\right| \leq C_{0}\left\|\mathcal{M}_{\mathcal{L}} f\right\|_{L^{1}\left(\mathbb{R}^{d}\right)},
$$

where the $\widetilde{a}_{j}$ are $(1,2,1)$-atoms.

Let $\widetilde{a}$ be a $(1,2,1)$-atom. By definition there is a function $\widetilde{b}$ and a ball $B=$ $B\left(x_{0}, r_{B}\right)$ such that

$$
\widetilde{a}=\mathcal{L} \widetilde{b}, \quad \operatorname{supp} \widetilde{b} \subset B\left(x_{0}, r_{B}\right), \quad \text { and } \quad\left\|\mathcal{L}^{k} \widetilde{b}\right\|_{L^{2}\left(\mathbb{R}^{d}\right)} \leq r_{B}^{2-2 k}|B|^{-1 / 2}, \quad k=0,1 .
$$

Set

$$
\mathbf{g}=r_{B}^{-2} \varphi\left(r_{B}^{2} \mathcal{L}\right) \widetilde{b}
$$

where $\varphi(\lambda)=\lambda e^{-\lambda}\left(1-e^{-\lambda}\right)^{-1}$. Obviously, $\varphi \in \mathcal{S}([0, \infty))$ and $\widetilde{a}=\left(I-T_{r_{B}^{2}}\right)(\widetilde{a}+\mathbf{g})$. By [14] the integral kernel $\varphi\left(r_{B}^{2} \mathcal{L}\right)(x, y)$ of the operator $\varphi\left(r_{B}^{2} \mathcal{L}\right)$ satisfies

$$
\left|\varphi\left(r_{B}^{2} \mathcal{L}\right)(x, y)\right| \leq c_{N} r_{B}^{-d}\left(1+\frac{|x-y|}{r_{B}}\right)^{-N} .
$$


Hence,

$$
|\mathbf{g}(x)| \leq C_{N} r_{B}^{-d}\left(1+\frac{\left|x-x_{0}\right|}{r_{B}}\right)^{-N}
$$

One can easily conclude from (6.7) that $\mathbf{g}$ can be written as

$$
\mathbf{g}=\sum_{i} \mu_{i} \mathbf{b}_{i}, \quad \sum_{i}\left|\mu_{i}\right| \leq C_{0}^{\prime}
$$

where each $\mathbf{b}_{i}$ is supported by a ball $B_{i}=B\left(y_{i}, r_{B}\right),\left\|\mathbf{b}_{i}\right\|_{L^{\infty}} \leq\left|B_{i}\right|^{-1}$ and the constant $C_{0}^{\prime}$ is independent of $\widetilde{a}$. Now let $A=\left\{x \in B:|\widetilde{a}(x)|>\varepsilon^{-1}|B|^{-1}\right\}$, where $\varepsilon>0$ is a small constant which will be determined later. By the Tchebychev inequality, $|A| \leq \varepsilon^{2}|B|$. Set $a^{0}=\widetilde{a}(x) \chi_{B \backslash A}(x)$ and $a^{\infty}(x)=\widetilde{a}(x) \chi_{A}(x)$. We have that $\left\|a^{0}\right\|_{L^{\infty}} \leq \varepsilon^{-1}|B|^{-1}$ and $\left\|a^{\infty}\right\|_{L^{3 / 2}\left(\mathbb{R}^{d}\right)} \leq \varepsilon^{1 / 3}|B|^{-1 / 3}$. Proposition 6.1 implies that $\left\|\mathcal{M}_{L}\left(I-T_{r_{B}^{2}}\right) a^{\infty}\right\|_{L^{1}} \leq C_{3 / 2} \varepsilon^{1 / 3}$. Thus, using (6.8), we get

$$
\widetilde{a}=\left(\left(I-T_{r_{B}^{2}}\right) a^{0}+\sum_{i} \mu_{i}\left(I-T_{r_{B}^{2}}\right) \widetilde{\mathbf{b}}_{i}\right)+\left(I-T_{r_{B}^{2}}\right) a^{\infty}:=\mathbf{h}_{1}+\mathbf{h}_{2},
$$

with

$$
\left\|\mathbf{h}_{1}\right\|_{\mathbf{H}_{\infty, \mathcal{L}, \text { at }}^{1}} \leq \max \left(\varepsilon^{-1}, C_{0}^{\prime}\right), \quad\left\|\mathcal{M}_{\mathcal{L}} \mathbf{h}_{2}\right\|_{L^{1}\left(\mathbb{R}^{d}\right)} \leq C_{3 / 2} \varepsilon^{1 / 3} .
$$

Thanks to (6.5) and (6.9), we decompose

$$
f=\mathbf{f}_{1}+f_{1}
$$

with

$$
\begin{aligned}
\left\|\mathbf{f}_{1}\right\|_{\mathbf{H}_{\infty, \mathcal{L}, \text { at }}^{1}} & \leq C_{0} \max \left(\varepsilon^{-1}, C_{0}^{\prime}\right)\left\|\mathcal{M}_{\mathcal{L}} f\right\|_{L^{1}\left(\mathbb{R}^{d}\right)}, \\
\left\|\mathcal{M}_{\mathcal{L}} f_{1}\right\|_{L^{1}\left(\mathbb{R}^{d}\right)} & \leq C_{0} C_{3 / 2} \varepsilon^{1 / 3}\left\|\mathcal{M}_{\mathcal{L}} f\right\|_{L^{1}\left(\mathbb{R}^{d}\right)} .
\end{aligned}
$$

Taking $\varepsilon$ small enough we guarantee that $C_{0} C_{3 / 2} \varepsilon^{1 / 3}<1 / 2$. Now we use the decomposition (6.10) for $f_{1}$ instead of $f$ and obtain $f_{1}=\mathbf{f}_{2}+f_{2}$ with

$$
\begin{aligned}
\left\|\mathbf{f}_{2}\right\|_{\mathbf{H}_{\infty, \mathcal{L}, \text { at }}^{1}} & \leq C_{0} \max \left(\varepsilon^{-1}, C_{0}^{\prime}\right)\left\|\mathcal{M}_{\mathcal{L}} f_{1}\right\|_{L^{1}\left(\mathbb{R}^{d}\right)} \\
& \leq C_{0} \max \left(\varepsilon^{-1}, C_{0}^{\prime}\right) 2^{-1}\left\|\mathcal{M}_{\mathcal{L}} f\right\|_{L^{1}\left(\mathbb{R}^{d}\right)} \\
\left\|\mathcal{M}_{\mathcal{L}} f_{2}\right\|_{L^{1}\left(\mathbb{R}^{d}\right)} & \leq C_{0} C_{3 / 2} \varepsilon^{1 / 3}\left\|\mathcal{M}_{\mathcal{L}} f_{1}\right\|_{L^{1}\left(\mathbb{R}^{d}\right)} \leq 2^{-2}\left\|\mathcal{M}_{\mathcal{L}} f\right\|_{L^{1}\left(\mathbb{R}^{d}\right)}
\end{aligned}
$$

Iterating this procedure we get the desired decomposition (6.4).

Proof of (6.1). According to Lemma 6.2 it suffices to prove that every generalized $(1, \infty)$-atom $\mathbf{a}=\left(I-T_{r^{2}}\right) \mathbf{b}$ can be written as

$$
\mathbf{a}=\sum_{j} \lambda_{j} b_{j}
$$

where the $b_{j}$ are $H_{w}^{1}$, at -atoms and $\sum_{j}\left|\lambda_{j}\right| \leq C$. 
Let $B=B\left(x_{0}, r\right)$ be a ball such that $\operatorname{supp} \mathbf{b} \subset B,\|\mathbf{b}\|_{L^{\infty}} \leq|B|^{-1}$. Then

$$
\int_{\mathbb{R}^{2}} \mathbf{a}(x) w(x) d x=\int_{\mathbb{R}^{2}} \mathbf{b}(x)\left(I-T_{r^{2}}\right) w(x) d x=0,
$$

because $w$ is $L$-harmonic. Clearly,

$$
|\mathbf{a}(x)| \leq C r^{-2} \exp \left(-\left|x-x_{0}\right| / r\right) .
$$

Write $2^{k} B=B\left(x_{0}, 2^{k} r\right)$ and set

$$
\begin{aligned}
& c_{k}=-w\left(2^{k} B\right)^{-1} \int_{\left(2^{k} B\right)^{c}} \mathbf{a}(x) w(x) d x, \quad k=0,1,2 \ldots ; \\
& b_{0}=\left(\mathbf{a}-c_{0}\right) \chi_{B}, \quad b_{k}=c_{k-1} \chi_{2^{k-1} B}-c_{k} \chi_{2^{k} B}+\mathbf{a} \chi_{2^{k} B \backslash 2^{k-1} B} .
\end{aligned}
$$

Then $\mathbf{a}=\sum_{j=0}^{\infty} b_{j}$. One can easily see, using the fact that $w(x) d x$ is a doubling measure, that each $b_{j}$ is a multiple of an $H_{w}^{1}$, at -atom and $\left\|b_{j}\right\|_{H_{w, \text { at }}^{1}} \leq C j^{-2}$, which finishes the proof.

\section{References}

[1] Auscher, P., Duong, X. T. and McIntosh, A.: Boundedness of Banach space valued singular integral operators and Hardy spaces. Unpublished preprint, 2005.

[2] Bernicot, F. And Zhao, J.: New abstract Hardy spaces. J. Funct. Anal. 255 (2008), no. 7, 1761-1796.

[3] Colfman, R.: A real variable characterization of $H^{p}$. Studia Math. 51 (1974), 269-274.

[4] Czaja, W. And Zienkiewicz, J.: Atomic characterization of the Hardy space $H_{L}^{1}(\mathbb{R})$ of one-dimensional Schrödinger operators with nonnegative potentials. Proc. Amer. Math. Soc. 136 (2008), no. 1, 89-94.

[5] Davies, E. B.: One-parameter semigroups. London Mathematical Society Monographs 15, Academic Press, London-New York, 1980.

[6] Duong, X. T. And Yan, L. X.: Duality of Hardy and BMO spaces associated with operators with heat kernel bounds. J. Amer. Math. Soc. 18 (2005), no. 4, 943-973.

[7] Dziubański, J., Garrigós, G., Martínez, T., Torrea, J. L. And ZienkieWICZ, J.: BMO spaces related to Schrödinger operators with potentials satisfying a reverse Hölder inequality. Math. Z. 249 (2005), no. 2, 329-356.

[8] Dziubański, J. And Zienkiewicz, J.: Hardy space $H^{1}$ associated to Schrödinger operator with potential satisfying reverse Hölder inequality. Rev. Mat. Iberoamericana 15 (1999), no. 2, 279-296.

[9] Dziubański, J. And Zienkiewicz, J.: Hardy spaces $H^{1}$ for Schrödinger operators with certain potentials. Studia Math. 164 (2004), no. 1, 39-53.

[10] Dziubański, J. And Zienkiewicz, J.: Hardy spaces $H^{1}$ for Schrödinger operators with compactly supported potentials. Ann. Mat. Pura Appl. (4) 184 (2005), no. 3, $315-326$. 
[11] Fefferman, C. And Stein, E. M.: $H^{p}$ spaces of several variables. Acta Math. 129 (1972), no. 3-4, 137-193.

[12] García-Cuerva, J. And Rubio de Francia, J. L.: Weighted norm inequalities and related topics. North-Holland Mathematics Studies 116, North-Holland, Amsterdam, 1985.

[13] Goldberg, D.: A local version of real Hardy spaces. Duke Math. J. 46 (1979), no. $1,27-42$.

[14] Hebisch, W.: A multiplier theorem for Schrödinger operators. Colloq. Math. 60/61 (1990), no. 2, 659-664.

[15] Hofmann, S., Lu, G. Z., Mitrea, D., Mitrea, M. and Yan, L. X.: Hardy spaces associated to nonnegative self-adjoint operators satisfying Davies-Gafney estimates. Mem. Amer. Math. Soc. 214 (2011), no. 1007.

[16] Simon, B.: Functional integration and quantum physics. Second Edition. AMS Chelsea Publishing, Providence, RI, 2005.

[17] Stein, E. M.: Harmonic analysis: real-variable methods, orthogonality, and oscillatory integrals. Princeton Mathematical Series 43, Monographs in Harmonic Analysis III, Princeton University Press, Princeton, NJ, 1993.

Received November 22, 2010; revised August 2, 2011.

JACEK DziUbański: Instytut Matematyczny, Uniwersytet Wrocławski, 50-384 Wrocław, Pl. Grunwaldzki 2/4, Poland.

E-mail: jdziuban@math.uni.wroc.pl

JACEK Zienkiewicz: Instytut Matematyczny, Uniwersytet Wrocławski, 50-384 Wrocław, Pl. Grunwaldzki 2/4, Poland.

E-mail: zenek@math.uni.wroc.pl

This research was supported by the Polish Ministry of Science and Higher Education, grant no. N N201-397137. 